\title{
Compassion fatigue and compassion satisfaction among palliative care health providers: a scoping review
}

\author{
Manal Hassan Baqeas ${ }^{*}$ (D), Jenny Davis and Beverley Copnell
}

\begin{abstract}
Background: Palliative care can be demanding and stressful for providers. There is increasing recognition in the literature of the impact of caregiving in palliative care settings, including compassion fatigue and compassion satisfaction. However, to date this literature has not been systematically reviewed. The purpose of this scoping review was to map the literature on compassion fatigue and compassion satisfaction among palliative care health providers caring for adult patients.

Methods: Scoping review method guided by Joanna Briggs Institute guidelines was conducted using four electronic databases to identify the relevant studies published with no time limit. Following the title and abstract review, two reviewers independently screened full-text articles, and extracted study data. A narrative approach to synthesizing the literature was used.

Results: Twenty studies were included in the review. Five themes emerged from synthesis: conceptualisation of compassion fatigue and compassion satisfaction; measurement of compassion fatigue and compassion satisfaction; consequences of compassion fatigue or compassion satisfaction and providing care for patients with life-threatening conditions; predictors or associated factors of compassion fatigue and compassion satisfaction among palliative care health providers; and strategies or interventions to support palliative care health providers and reduce compassion fatigue.

Conclusions: Limited studies examined the effectiveness of specific interventions to improve compassion satisfaction and reduce compassion fatigue among palliative care health providers. Further investigation of the impacts of compassion fatigue and compassion satisfaction on palliative care health providers and their work is also needed.
\end{abstract}

Keywords: Compassion fatigue, Compassion satisfaction, Palliative care, Palliative care health providers, Scoping review

\section{Background}

Palliative care aims to support people with life-threatening conditions and improve their quality of life [1]. Palliative care health providers (PCHP) comprise medical, nursing, and allied health care professionals who work in palliative care settings and who have specific knowledge, skills, and expertise in providing care for people living with a life limiting illness and their families. PCHP can provide direct

\footnotetext{
* Correspondence: M.Baqeas@latrobe.edu.au

School of Nursing and Midwifery, La Trobe University, Bundoora, VIC 3086, Australia
}

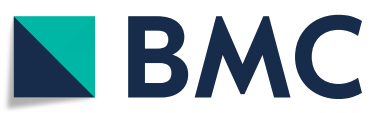

(c) The Author(s). 2021 Open Access This article is licensed under a Creative Commons Attribution 4.0 International License, which permits use, sharing, adaptation, distribution and reproduction in any medium or format, as long as you give appropriate credit to the original author(s) and the source, provide a link to the Creative Commons licence, and indicate if changes were made. The images or other third party material in this article are included in the article's Creative Commons licence, unless indicated otherwise in a credit line to the material. If material is not included in the article's Creative Commons licence and your intended use is not permitted by statutory regulation or exceeds the permitted use, you will need to obtain permission directly from the copyright holder. To view a copy of this licence, visit http://creativecommons.org/licenses/by/4.0/. The Creative Commons Public Domain Dedication waiver (http://creativecommons.org/publicdomain/zero/1.0/) applies to the data made available in this article, unless otherwise stated in a credit line to the data.

care in various settings such as dedicated hospital wards, hospices, and community, and through consultancy to patients in other areas [2].

Prolonged contact with these patients predisposes PCHP to emotional and psychological distress such as compassion fatigue. There are various definitions of compassion fatigue documented in the literature. In general, compassion fatigue is a term used to describe the exhaustion that results from prolonged exposure to compassion stress among those who work in a caring profession [3]. Compassion fatigue is also described as 
the diminished ability to feel compassion or empathize when providing care. In contrast, compassion satisfaction is related to the pleasure derived from alleviation of patient suffering and positive work experience [4]. There is no consensus in the literature on the dimensions or components of compassion fatigue. However, there is a general agreement that compassion fatigue is related to both burnout (BO) and secondary traumatic stress (STS). While STS is very closely related to compassion fatigue, the nature of the relationship is defined differently and both terms used interchangeably by some authors [4]. The concept of compassion satisfaction is related to positive work experience, whereas compassion fatigue is associated with physical and emotional exhaustion, caused by constant, progressive, and cumulative negative experiences associated with various clinical settings $[3,5,6]$. Compassion fatigue has negative impacts on job satisfaction and patient outcomes [7-9]. This emphasizes the significance of investigating compassion fatigue in PCHP.

To date, compassion fatigue has been widely studied in health care providers in a range of settings, as synthesized in a recent meta-narrative review [10]. However, to our knowledge, no such synthesis has been undertaken of literature pertaining specifically to PCHP. This gap in the literature makes it difficult to identify and implement interventions to support these workers. Therefore, the aim of this scoping review is to synthesize findings from extant research about compassion fatigue and compassion satisfaction among PCHP.

\section{Methods}

The scoping review, as a method, is suitable when the study topic is abstract, broad, emerging, or multidimensional [11]. Scoping reviews are used to answer a broad question such as "what is known about the study concepts?" [11]. It was, therefore, deemed suitable to address the aim of the current study. It answers the research question through a narrative synthesis of the literature. In addition, it is used to summarize the current knowledge about a topic and identify knowledge gaps regardless of the quality of reviewed studies and their design [11].

The current scoping review was conducted based on the guidelines published by the Joanna Briggs Institute (JBI) [12]. These guidelines were developed based on the previous work by Arksey and O'Malley [13] and Levac, Colquhoun, and O'Brien [14]. In addition, the literature review followed the PRISMA-ScR checklist to provide clear details of the search protocol and enhance methodological transparency [11]. As per the Joanna Briggs Institute guidelines, the following five stages were followed: 1 . Identifying the research question 2 . Identifying relevant studies 3 . Selection of relevant studies 4 .
Charting the data 5. Collating, summarizing and reporting the results [12]. There is a sixth (optional) step that includes consultation with key stakeholders. This step was omitted, however, and only evidence published in peer-reviewed literature was included.

\section{Stage 1. Identifying the research question}

This review aims to identify what is known about compassion fatigue and compassion satisfaction among PCHP. To address the study aim, the review was conducted to answer the following question: "what research has been undertaken on compassion fatigue and compassion satisfaction among palliative care health providers?"

\section{Stage 2. Identifying relevant publications}

The review was conducted by a team of researchers including the primary researcher, content experts, and methodological experts. A search of four electronic databases: MEDLINE (OVID), CINAHL, PsycInfo, and EMBASE was conducted in August 2019. To ensure a comprehensive search, the search terms "compassion fatigue", "compassion satisfaction", and "palliative care health providers" were initially kept broad and then exploded to cover MeSH terms. In addition, keywords included in the title and abstract of retrieved papers, and the keywords used to describe the articles were identified. These keywords were searched across the databases. Finally, the reference lists of the selected articles were hand searched to identify additional studies. The terms "compassion fatigue", "compassion satisfaction", and "palliative care health providers" were combined with the following terms: "burnout, professional", "stress disorders, post-traumatic", "fatigue, compassion", "secondary trauma", "secondary traumatic stress", "secondary traumatization", "trauma, vicarious", "traumas, secondary", "traumatic stress, secondary", "burnout, career', "burnout, occupational", "burnout, professional", "secondary post-traumatic stress", "hospice professionals", "hospice, palliative care nursing", "palliative care", "palliative medicine", "terminal care", "palliative supportive care", and "palliative treatment". The Boolean operators 'AND' and 'OR' were used to combine various terms and concepts. All identified sources were stored in the EndNote reference program. Irrelevant records and duplicates were excluded from the literature search. The final screening of title/abstract and then full text was managed in Covidence.

Inclusion criteria were: 1. all research designs (e.g., quantitative, qualitative, mixed methods, and systematic reviews); 2. addressing compassion fatigue and compassion satisfaction from the perspectives of PCHP caring for adult patients in any practice setting; 3 . published in English with no date limits applied. Exclusion criteria 
were: 1. grey literature (e.g., book chapters, theses, reports, and conference abstracts); 2 . Non-research publications (eg editorials; discussion papers; opinion pieces); 3. targeting volunteers working in palliative care settings; 4. investigating $\mathrm{BO}$ without STS or Compassion Fatigue; 5. focusing on PCHP working with pediatric patients as we consider pediatric palliative care has distinct differences from adult palliative care and can be considered a speciality in its own right [15].

\section{Stage 3. Publication selection}

After removal of duplicates, article titles and abstracts were screened by two researchers independently. Disagreements were discussed and resolved by consensus among the research team. After full text screening, studies meeting all inclusion criteria were included in the final review.

\section{Stage 4. Charting the data}

A data extraction table was used to extract the data from the included studies. Extracted data included country, year of publication, names of authors, study purpose, research design, study sample, and main study findings. The data extraction was conducted by one researcher and reviewed by the research team. Any disagreements in data extraction were resolved by consensus. References were managed utilising EndNote (version X9) and included studies were imported to Covidence during the final screening. In line with the PRISMA-ScR standards [11], no formal quality appraisal was undertaken as it was not intended to exclude any paper based on quality assessment.

\section{Stage 5 data synthesis}

Narrative synthesis was employed due to the heterogeneity of the studies. The characteristics of the reviewed studies (i.e design, sample, settings, main variables, and publication year) were collated and summarized. Studies were summarized in a Table and a content analysis was performed based on the tabulated data. Then, contents were translated into main themes. Lastly, the findings were interpreted and compared with studies from other settings.

\section{Results}

Overall, the initial search yielded 1822 records. After removing duplicates, 1085 records were screened for potential relevance by title and abstract. Of these, 921 records were found to be irrelevant and 164 full-text articles were screened. Finally, 144 articles were excluded and 20 articles were included in the final review (Fig. 1). Studies were conducted in different countries worldwide, the majority in a Western setting. Countries represented were: United States $(n=9)$, Spain $(n=3)$, Israel $(n=3)$, with one study from each of Australia, Canada, New Zealand, and India. The majority of the studies were published within the last 5 years $(n=15)$. More than half of the studies were correlational $(n=10)$, four studies were qualitative, one a quantitative descriptive study, one a pre-post study with control group, one pre-post with no control group, two studies examined the psychometric properties of the Professional Quality of Life (ProQOL) scale, one paper was a systematic review. Study populations included PCHP from several disciplines $(n=14)$, only nurses $(n=3)$, or only physicians $(n=2)$.

The samples in the included studies were recruited from various settings that provide palliative care (Table 1). One study was conducted in inpatient hospices and hospitals [20]. One study was conducted in inpatient hospices [21], one in outpatient hospices [1], and one in hospice settings without specifying whether inpatient or outpatient [22]. One study was conducted in outpatient palliative care setting [24]. Eight studies included participants from both inpatient and outpatient settings including hospices $[16,18$, 19, 23, 28-30, 33]. However, the combination between inpatient and outpatient settings in these eight studies was unclear and not described in detail. Six studies included participants from settings that provide inpatient and outpatient services without stating specifically if all participants were recruited from inpatient, outpatient, or both [17, 25-27, 31, 32].

\section{Themes extracted from the included studies}

Five main themes were identified in the synthesis of the included studies: 1 . conceptualisation of compassion fatigue and compassion satisfaction; 2. measurement of compassion fatigue and satisfaction; 3 . consequences of compassion fatigue or satisfaction and of providing care for patients with life-threatening conditions; 4. predictors or associated factors of compassion fatigue and satisfaction among PCHP; 5. strategies or interventions to support PCHP and reduce compassion fatigue. These themes are described further in the following sections. The summary of the included studies is shown in Table 1.

\section{Theme 1- conceptualisation of compassion fatigue}

Overall, the reviewed studies did not discuss the conceptualisation of compassion fatigue in depth. Their definition was mainly embedded in that used by the measurement tool and thus reflects changes in the concept over time. Compassion satisfaction was defined by some studies as a positive consequence of providing care for acutely ill or traumatised patients (e.g., a sense of accomplishment and reward) $[1,18,19,22,25,30,31]$. Some studies treated compassion fatigue as a single discrete entity with no constitutive components $[1,21]$. 


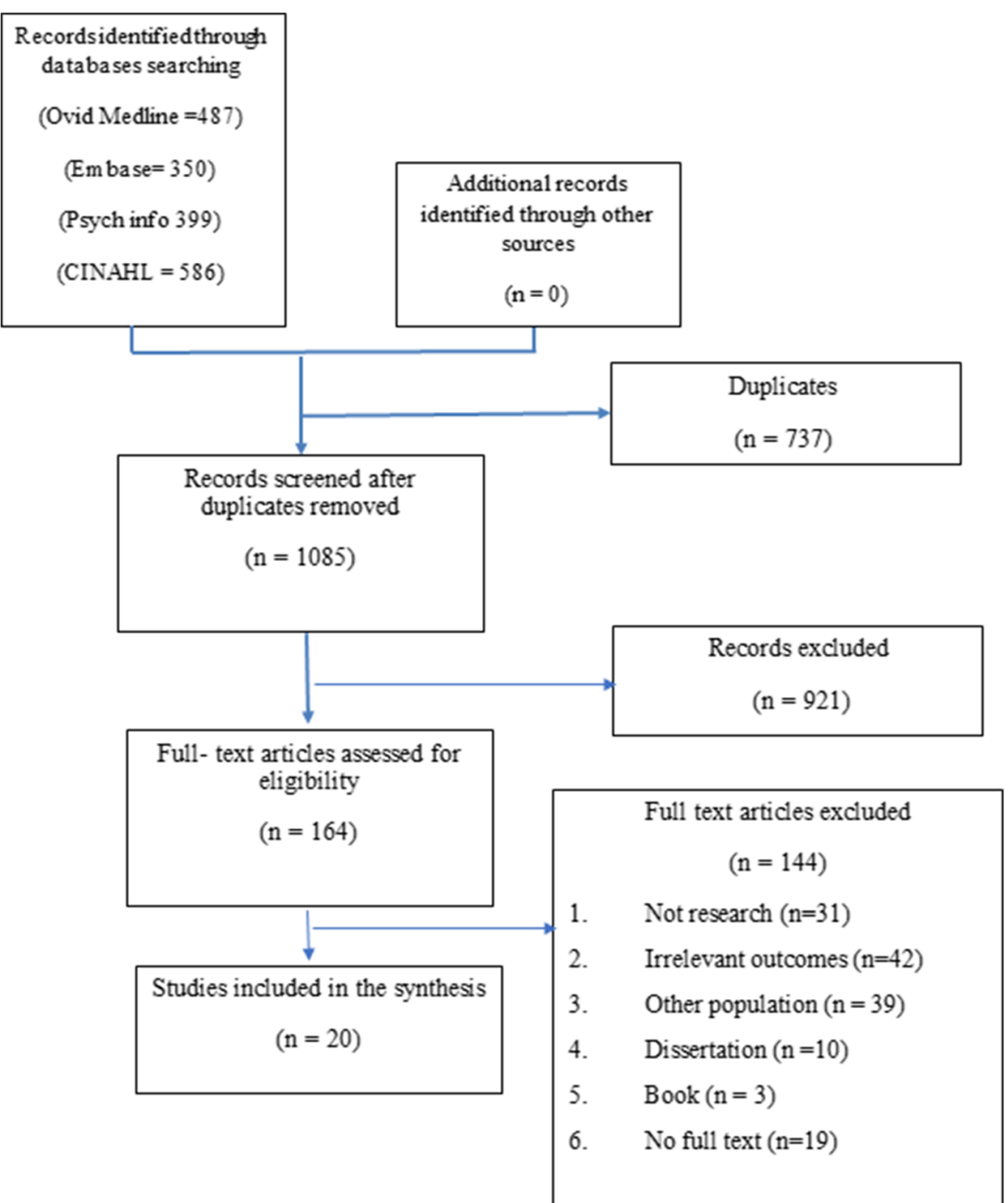

Fig. 1 Flow diagram of search strategy

On the other hand, some studies treated compassion fatigue as being synonymous with STS, and these terms were used interchangeably [20,31, 32]. The remaining studies conceptualized compassion fatigue as having two discrete components (STS and BO), each of which was measured separately $[16,18,19,22,23,25,27-30]$. The qualitative studies did not specify a clear definition of compassion fatigue [17, 24, 26, 33].

\section{Theme 2: measurement of compassion fatigue and satisfaction}

The measurement tools used to assess compassion fatigue and compassion satisfaction among PCHP included the 30-item professional quality of life scale (ProQOL) scale, the 20-item compassion fatigue scale (CFS), and the 13-item Compassion Fatigue Short-Scale. The various versions of the ProQOL reflect the changes in conceptualisation described in the previous section. The ProQOL-V includes two domains of compassion fatigue (composed of $\mathrm{BO}$ and STS) and compassion satisfaction. The ProQOL-IV measures three domains: compassion satisfaction, $\mathrm{BO}$, and compassion fatigue/ secondary trauma. The ProQOL-III measures three domains: compassion satisfaction, $\mathrm{BO}$, and compassion fatigue. The 20-item compassion fatigue scale (CFS) is a subscale of the 66-item Compassion Satisfaction/Fatigue Self-Test for Helpers which measures compassion satisfaction, compassion fatigue, and $\mathrm{BO}$. The 13-item Compassion Fatigue Short-Scale measures compassion fatigue in two dimensions (secondary trauma and job $\mathrm{BO}$ ).

The most commonly used measure of compassion fatigue and compassion satisfaction among PCHP was the ProQOL scale (III, IV, and V versions), which was used 


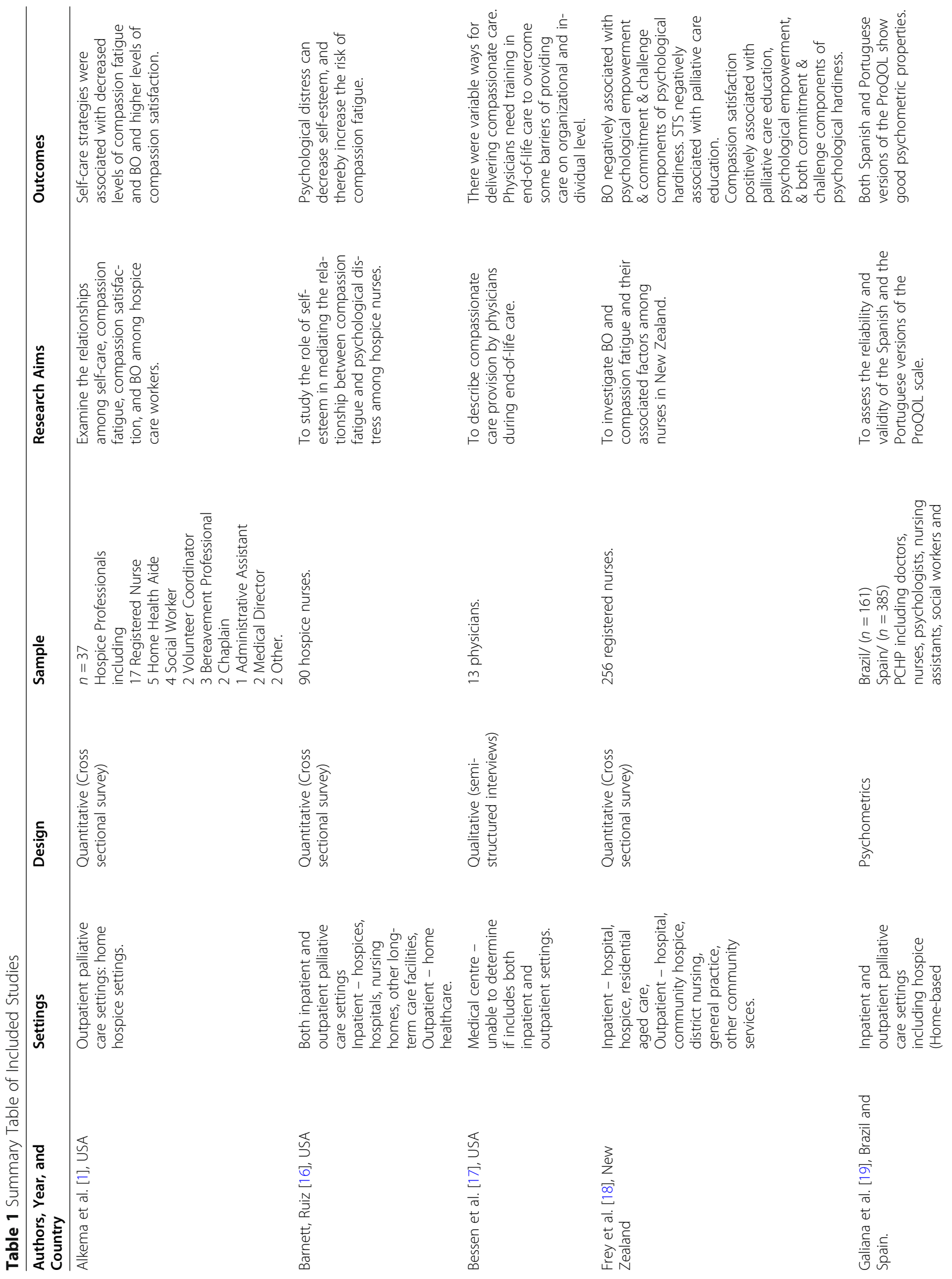




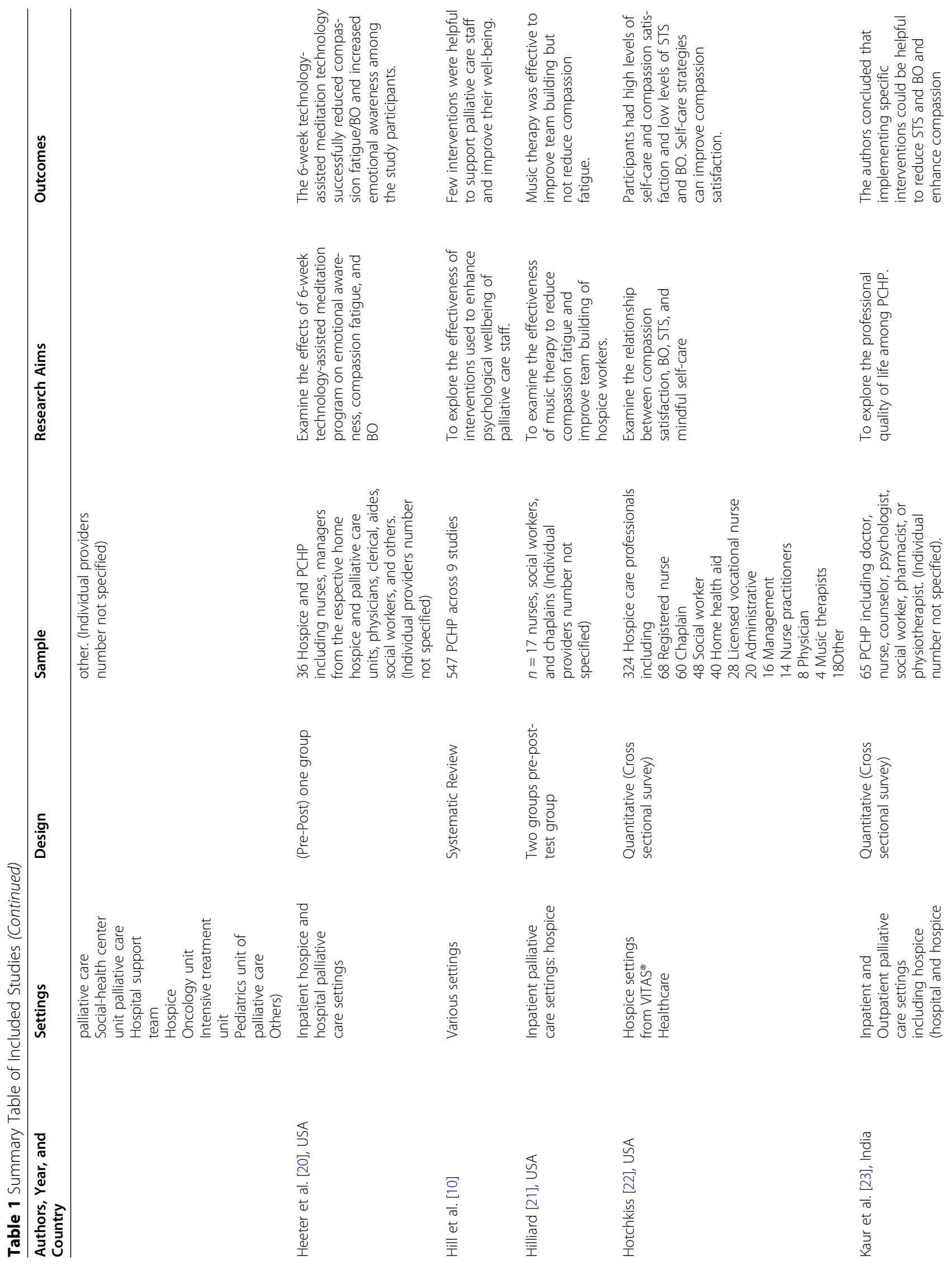




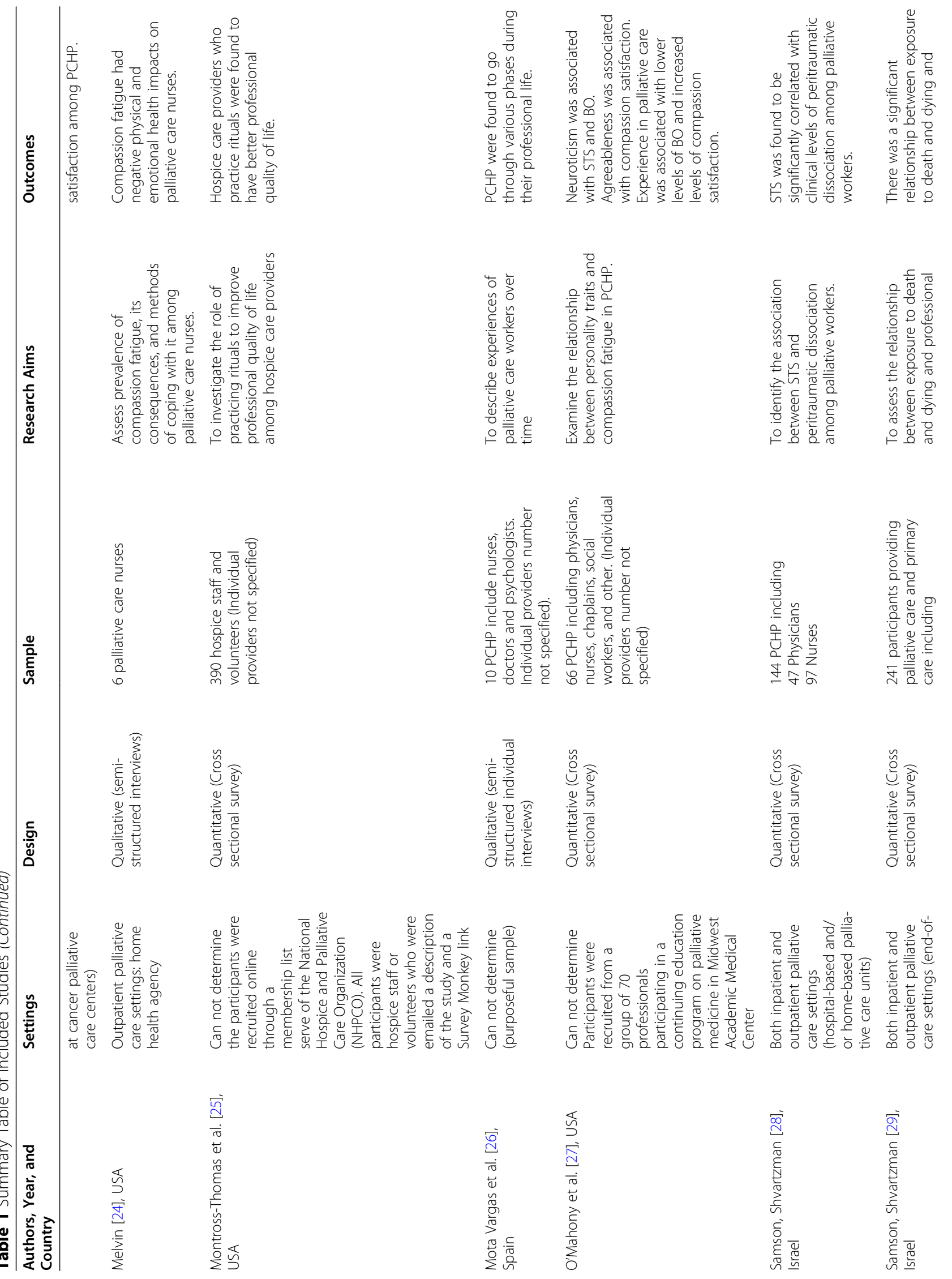




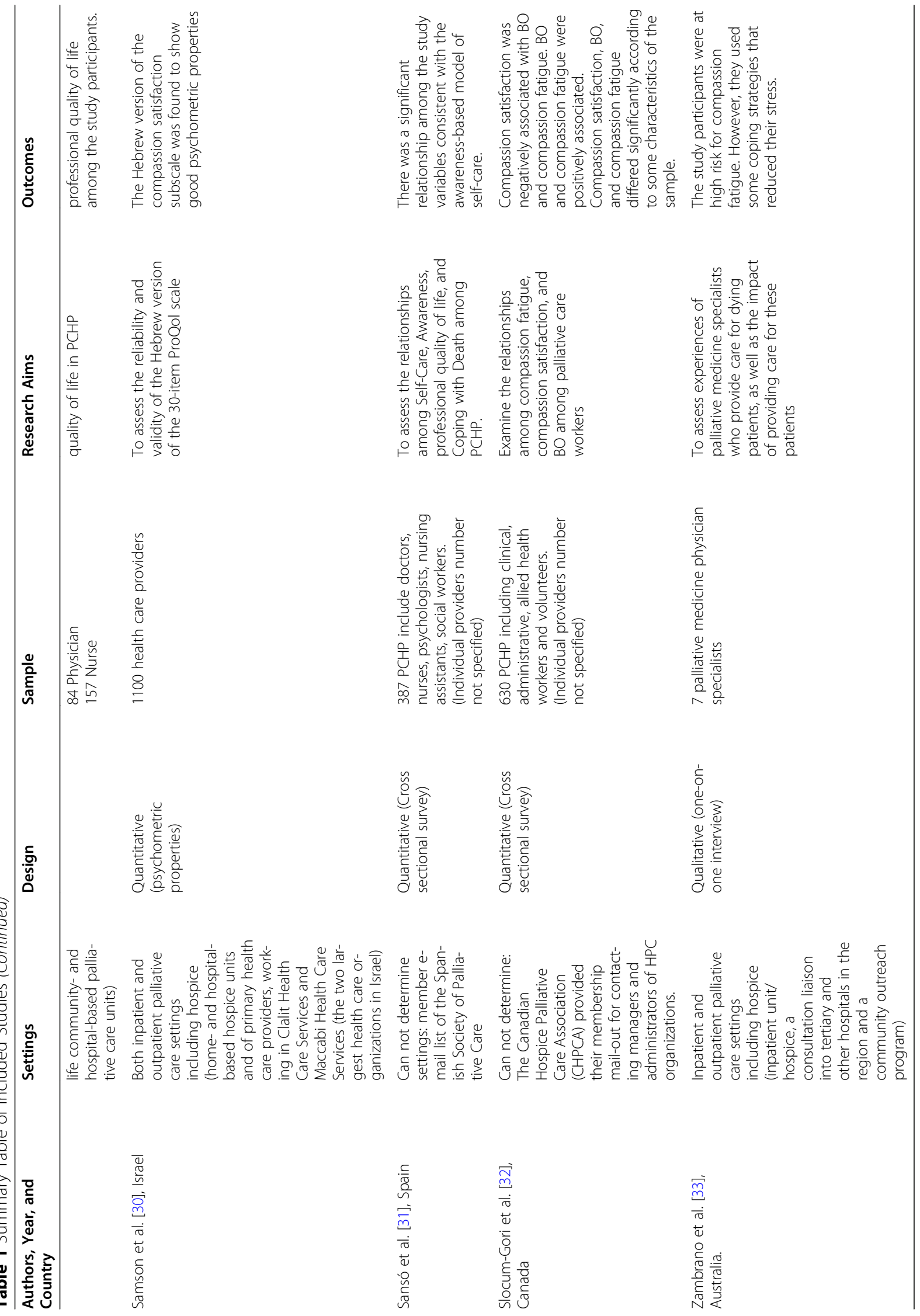


in 11 studies [1, 18, 20, 22, 23, 25, 27-29, 31, 32]. This scale measures compassion satisfaction, STS, and BO. The items of each subscale are rated on a five-point Likert-type scale. The scale has demonstrated excellent psychometric properties with Cronbach's alpha of 0.80 or more for its subscales [4].

The Compassion Fatigue Scale (CFS) was used in only one study [21] which was a pre-post study. This tool is distinguished from the other tools by focusing more on the helper and working environment. In addition, the Compassion Fatigue Short-Scale was used in one study [16]. This tool measures only compassion fatigue. Both the 20-item CFS and the 13-item CFS were reported to have adequate reliability and validity $[16,21]$. Therefore, all of the three tools have been utilised internationally with various populations. Apart from the psychometric properties of these three measurement tools, authors did not report any other evidence about their efficacy. In addition, they did not provide a rationale for their choice of these tools in their studies.

Four studies reported the levels of compassion fatigue and compassion satisfaction among PCHP. All four used the professional quality of life scale (ProQOL) scale. In the study of Frey et al., [18] about half (48.4\%) of palliative care nurses had moderate to high levels of compassion satisfaction. However, about a quarter of the participants had high BO scores (26.8\%) and more than half (51.6\%) had moderate STS [18]. O'Mahony et al. [27] found that palliative medicine physicians had overall high levels of compassion satisfaction and low levels of BO and STS. Alkema, Linton, and Davies [1] found that the mean scores of compassion satisfaction, $\mathrm{BO}$, and compassion fatigue among hospice professionals were in the average range. Finally, Kaur, Sharma, and Chaturvedi reported that, among palliative care providers, $49.2 \%$ had an average level of compassion satisfaction, $53.8 \%$ had an average level of BO, while $95.4 \%$ scored above 75 th percentile on STS [23].

\section{Theme 3: consequences of compassion fatigue}

Two studies, both qualitative, reported consequences of compassion fatigue among their findings. A study conducted by Melvin reported that providing palliative care and working with dying patients could contribute to compassion fatigue among PCHP [24]. The author also suggests that providing palliative care and working with dying patients could contribute to physical and emotional consequences. PCHP reported feeling responsible for patient care even after going home and leaving the workplace [24]. In addition to compassion fatigue, working with dying patients likely affects many dimensions concerning mental health including feelings of guilt, sadness, crying, thinking of death, remembering personal experiences with death, isolation, and grief [33].

\section{Theme 4: predictors or associated factors of compassion} fatigue and satisfaction

Eleven articles provided data about the correlates of high levels of compassion fatigue and poor compassion satisfaction among PCHP. In general, studies included PCHP from several disciplines. However, two studies had only nurse samples and one study had both physician and nurse samples. The synthesis of these studies is included below.

In general, demographic, personal, and organisational factors were associated with compassion fatigue and compassion satisfaction among PCHP. Demographic factors were found to be associated with compassion fatigue in some studies. Slocum-Gori et al. [32] found that employment status was associated with compassion fatigue as part-time workers had lower scores than those who worked full time. Additionally, they found that greater experience in palliative care was associated with lower levels of BO. O'Mahony et al. [27] supported these results and found that duration of experience in palliative care was associated with higher levels of compassion satisfaction.

Personal factors were found to be associated with compassion fatigue in several studies. For example, having a neuroticism personality trait was associated with increased levels of STS and BO among PCHP, while having an agreeableness personality trait was associated with increased levels of compassion satisfaction [27]. In addition, psychological hardiness (e.g., commitment and challenge) were associated with lower $\mathrm{BO}$ and greater compassion satisfaction [18]. Furthermore, practicing some personal rituals on specific occasions was associated with lower $\mathrm{BO}$ and more compassion satisfaction among hospice staff [25]. Also, the ability to cope with death was associated with lower levels of compassion fatigue and $\mathrm{BO}$ and higher compassion satisfaction among PCHP [31]. Greater exposure to death was also significantly correlated with STS among physicians and nurses employed in a palliative care unit [29]. In addition, high levels of dissociation (detachment) were associated with higher levels of STS [28]. Psychological distress was also associated with increased compassion fatigue [16]. Further, using self-care strategies was associated with lower levels of compassion fatigue and BO and higher levels of compassion satisfaction [1]. Further, mindful self-care was associated with more compassion satisfaction and less risk of $\mathrm{BO}$ among health care workers in the palliative care setting [22].

Frey et al. found that organizational factors such as work-related empowerment could decrease BO levels [18]. Furthermore, the authors found that STS was negatively associated with previous palliative care education [18]. Kaur et al. concluded that receiving training in palliative care was associated with lower levels of $\mathrm{BO}$ and 
STS [23]. Kaur et al. found that professional orientation was associated with compassion satisfaction, with nurses scoring lower levels than other health professionals [23]. Slocum-Gori et al. [32] found that compassion fatigue was negatively correlated with compassion satisfaction and positively correlated with $\mathrm{BO}$.

\section{Theme 5: strategies or interventions to support PCHP or reduce compassion fatigue}

In one systematic review, Hill et al. identified multiple interventions reported to improve wellbeing of PCHP; however, most were found to be ineffective in reducing compassion fatigue [34]. Examples of these interventions include cognitive training, education, relaxation, and support [34]. Two of the included studies evaluated interventions to reduce compassion fatigue among PCHP. The first study by Heeter, Lehto, Allbritton, Day and Wiseman examined the effectiveness of a 6 -week meditation program delivered via smartphone apps to reduce compassion fatigue among 36 PCHP [20]. The single group pre and post-test study design reported a significant reduction in compassion fatigue after the intervention [20]. Another study conducted by Hilliard [21] investigated the effectiveness of a music therapy intervention to reduce compassion fatigue in a sample of 17 hospice workers. Participants were randomly assigned to an ecological music therapy group and a didactic music therapy group. A pre-and post-test was performed to measure compassion fatigue levels. The results indicated no significant differences in compassion fatigue between pre-and post-test scores of compassion fatigue in either group [21].

Four qualitative studies reported strategies to support PCHP from the perspectives of the study participants $[17,24,26,33]$. These studies did not actually measure the effectiveness of these strategies. However, the researchers interviewed PCHP and asked them to list strategies they believed helped to protect them from compassion fatigue. Palliative care nurses in the study by Melvin described adopting various strategies including setting professional boundaries, seeking support from colleagues and supervisors, reflection, physical exercise, and social activities out of work [24]. In the study of Mota Vargas et al. researchers interviewed PCHP and asked them to identify the self-care strategies they used [26]. Participants reported that reflecting on their experience of providing palliative care, understanding the methods used to enhance self-control, and acknowledging one's limits and accepting the fact that many things cannot be changed and learning to live with them were the most commonly used strategies. Other self-care strategies included attending training in palliative care, improving their communication skills, and developing personal hobbies [26]. Zambrano, Chur-Hansen, and
Crawford reported that PCHP highlighted supportive measures such as finding spiritual meaning, receiving support, and using both problem-focused and emotionfocused coping strategies [33]. Bessen, Jain, Brooks et al. reported that physicians described sharing experiences with their colleagues or using individual-based strategies (e.g., improving self-awareness) to prevent compassion fatigue [17].

\section{Discussion}

This scoping review mapped available evidence on compassion fatigue and compassion satisfaction among PCHP in various palliative care settings. The current scoping review included all relevant studies regardless of the publication year but the majority that met inclusion criteria were published within the last 5 years $(n=16)$. This suggests that interest in compassion fatigue and compassion satisfaction in the field of palliative care is increasing.

Themes that emerged in this review were also reported by previous reviews focusing on other health professionals in non-palliative care settings. In a metanarrative review related to compassion fatigue in health literature, the main themes that emerged were related to predictors/risk factors of compassion fatigue, its consequences, conceptualization, and measurement [10]. Another review related to compassion fatigue in cancer care providers included themes related to compassion fatigue prevalence, measurement, and management [35]. These reviews reported various predictors/risk factors and consequences of compassion fatigue that are, to some extent, similar to these reported in the current study.

Findings in our review suggest a general agreement that compassion satisfaction reflects a sense of accomplishment and reward of providing care for patients [1, $18,19,22,25,30,31]$. However, there was no consensus on the definition of compassion fatigue in palliative care settings. While some studies treated compassion fatigue as a single discrete entity, or synonymous with STS [20, 31,32 , it was considered a multi-dimensional concept by others $[16,18,19,22,23,25,27-30]$. The multidimensionality of compassion fatigue is further complicated because it is informed by different theories that inform the definition of compassion fatigue [10, 36]. This renders the development of a unified meaning of compassion fatigue difficult. This also resulted in the variability of the domains or subscales of the measures used to assess compassion fatigue. Most of the included studies used the ProQOL scale which assessed BO and STS as components of compassion fatigue rather than reporting an overall score for compassion fatigue.

Compassion is a central concept for PCHP who provide care for people with life limiting conditions. The 
more empathic a palliative care provider becomes, the more likely compassion fatigue will occur. Therefore, it is important to educate PCHP to modify empathetic ability in response to prolonged work with patients needing palliative care. The human nervous system plays an important role in regulating the empathetic response of the individual. Recent literature has shown that empathy is influenced by nervous system stimulation and it may lead to empathic distress [37].

The literature review revealed various organizational factors (e.g., work-related empowerment, receiving training in palliative care, and being recognized as a palliative care nurse) and demographic factors (e.g., employment status as part-time workers or full time and experience in palliative care) associated with compassion fatigue and compassion satisfaction across PCHP. Further, it was noted that some personal factors associated with compassion fatigue and compassion satisfaction were nonmodifiable (e.g., neuroticism personality trait and psychological hardiness). Additional factors included personal variables such as practicing some personal rituals, the ability to cope with death and self-care, levels of dissociation, using self-care strategies and mindful self-care. Therefore, it can be concluded that compassion fatigue and compassion satisfaction are predicted by many factors, some of which may not be modifiable.

The majority of studies included participants from multiple work settings (hospital, hospice and community) and none compared findings across settings or attempted to differentiate between them. Given that work in the various settings can vary considerably, the incidence and experience of compassion fatigue may also vary. Future research should explore the impact of work setting on compassion fatigue and compassion satisfaction.

Receiving palliative care training or education was found to help reduce the likelihood of developing symptoms of compassion fatigue [18, 23, 38]. None of these studies explored the content of education programs to identify which aspects induced this effect. Studies in nonpalliative care settings have investigated training programs specifically focused on reducing or preventing compassion fatigue. For example, in a Pre- Post- test study conducted to examine the effect of Mindful Self-Compassion (MSC) training on compassion fatigue and resilience among nurses working in various settings, there was a significant reduction in the scores of secondary trauma and $\mathrm{BO}$ after the intervention [39]. Another study reported a significant reduction in participants' compassion fatigue and $\mathrm{BO}$ and improvement in compassion satisfaction after Compassion Fatigue Specialist Training for mental health professionals [40]. It would seem likely given the nature of palliative care work that specialist education programs would include a focus on similar self-care activities; an examination of the curricula of these programs would be useful in explicating this content. We recommend that PCHP undergo specific education/training in this area, whether through formal programs or continuing professional development.

A number of interventions have been shown to reduce compassion fatigue and improve compassion satisfaction across a wide range of populations [39, 40]. However, few intervention studies were conducted specific to the field of palliative care. Only two of the included studies in this review involved interventions and measured their effectiveness to mitigate compassion fatigue and improve compassion satisfaction among PCHP. Only one of the tested interventions (The 6-week technology-assisted meditation) was found to be effective in reducing compassion fatigue. Despite this, many descriptive or correlational studies pointed to such interventions. Other studies investigated strategies to support PCHP using self-report data with correlational or qualitative approaches rather than actually implementing these strategies or measuring their effectiveness [17, 24, 26, 33]. Therefore, most of the knowledge regarding the interventions used to mitigate compassion fatigue and improve compassion satisfaction among PCHP is informed by low level evidence. Furthermore, while there is some overlap between palliative care and other health care specialties, there are also aspects that are unique to palliative care. Therefore, it cannot be assumed that research undertaken in other specialty areas can be applied to PCHP, and we recommend interventions be tested in this population.

\section{Strengths and limitations}

The strengths of this review include conducting a comprehensive search with no limits on publication dates. In addition, studies that used concepts related to compassion fatigue but did not examine the concept directly (e.g., empathy, moral distress) were excluded from the literature search to make the search methodology more rigorous. Nevertheless, the review has some limitations. First, some relevant studies may have been missed despite using a rigorous search strategy. This could occur due to the complexity of compassion fatigue terms and inconsistencies in its conceptualisation across different studies. Second, only publications written in English were included which limits generalisability and may introduce language bias. The limited number of studies examining compassion fatigue in palliative care settings may warrant conducting a broad search in all languages. Grey literature was excluded, which may introduce publication bias.

The results of this review highlight a gap in the literature examining impacts of compassion fatigue and compassion satisfaction on PCHP. This gap in the literature demonstrates the need for further research on the 
impacts of compassion fatigue and compassion satisfaction on PCHP. Therefore, as nurses make up a significant proportion of the palliative care health provider workforce, we recommend exploring the impact of compassion fatigue and compassion satisfaction on productivity among palliative care nurses. Targeting a homogeneous sample of nurses is also recommended since the included studies predominantly involved heterogenous samples of PCHP rather than specifically nurses. Research is also required to understand whether and how the experience of compassion fatigue and compassion satisfaction may vary across different work environments. In addition, there is a need to conduct interventional studies to identify the most effective strategies, including education or training, to reduce compassion fatigue among PCHP.

\section{Conclusion}

This review sought to identify current evidence about compassion fatigue and compassion satisfaction among PCHP. Most of the studies investigating the impacts of compassion fatigue and compassion satisfaction on PCHP were descriptive in nature. This indicates a gap in the literature that needs more investigation. Only one study identified an effective intervention to reduce compassion fatigue in PCHP. Most of the reviewed studies were correlational or exploratory in nature which affects the quality and strength of the retrieved evidence. One important aspect to be considered is the impact of compassion fatigue and compassion satisfaction on the productivity of PCHP and their ability to provide safe and compassionate care. This is an important topic especially among palliative care nurses since they are the largest group of PCHP and they spend a long time caring for people with life-threatening conditions and related trauma. The current work suggests a need to fill various gaps in knowledge and provides a clear direction for future research.

\section{Abbreviations}

PCHP: Palliative care health providers; BO: Burnout; STS: Secondary traumatic stress; ProQOL: Professional quality of life; CFS: Compassion fatique scale

\section{Acknowledgements}

We would like to thank Ms. Elizabeth Lawrence for her contributions to developing the search strategy for this manuscript.

\section{Authors' contributions}

$\mathrm{MB}$ and $\mathrm{BC}$ conceived the study. MB developed the search strategy, conducted the initial literature search, and contributed to the writing of the manuscript. BC and MB extracted and screened articles. BC and JD provided substantive expertise, oversaw all stages of the review, provided expertise to the review and analysis process, and critically revised the manuscript. All authors approved the final version of the manuscript.

\section{Funding}

MB was supported by a scholarship from the Ministry of Higher Education Saudi Arabia. The funding body had no involvement in the design or conduct of the study, nor in the writing of the manuscript. No other funding was received.

\section{Availability of data and materials}

Data used in this manuscript consist of published articles which cannot be shared by the authors for copyright reasons but are available through

subscription to the relevant journals/databases.

\section{Declarations}

Ethics approval and consent to participate

Not applicable; no original data.

\section{Consent for publication}

Not applicable; no details, images, or videos relating to individual persons included.

\section{Competing interests}

The author(s) declare no potential conflicts of interest with respect to the manuscript, authorship, and/or publication of this article.

Received: 8 August 2020 Accepted: 1 June 2021

Published online: 23 June 2021

\section{References}

1. Alkema K, Linton JM, Davies R. A study of the relationship between selfcare, compassion satisfaction, compassion fatigue, and burnout among hospice professionals. J Soc Work End-of-Life Palliat Care. 2008;4(2):101-19.

2. Consortium GRPC. Specialist palliative care for complex care and referral to specialist palliative care - Grampians Region Palliative Care Consortium. Grampians Region Palliative Care Consortium. [cited 13 December 2020]. Available from: https://grampianspalliativecare.com.au/health-careprofessionals/specialist-palliative-care-for-complex-care/

3. Sorenson C, Bolick B, Wright K, Hamilton R. Understanding compassion fatigue in healthcare providers: a review of current literature. J Nurs Scholarsh. 2016:48(5):456-65.

4. Stamm B. The concise ProQOL manual. 2nd ed. Pocatello: Beth Hundall Stamm; 2020. [cited 14 January 2021]. Available from: https://proqol.org/ uploads/ProQOLManual.pdf

5. Coetzee SK, Klopper HC. Compassion fatigue within nursing practice: a concept analysis. Nurs Health Sci. 2010;12(2):235-43.

6. Nolte AG, Downing C, Temane A, Hastings-Tolsma M. Compassion fatigue in nurses: a metasynthesis. J Clin Nurs. 2017;26(23-24):4364-78.

7. Drury V, Craigie M, Francis K, et al. Compassion satisfaction, compassion fatigue, anxiety, depression and stress in registered nurses in Australia: phase 2 results. J Nurs Manag. 2014;22(4):519-31.

8. Harris C, Griffin MTQ. Nursing on empty: compassion fatigue signs, symptoms, and system interventions. J Christ Nurs. 2015;32(2):80-7.

9. Hegney DG, Craigie M, Hemsworth D, et al. Compassion satisfaction, compassion fatigue, anxiety, depression and stress in registered nurses in Australia: study 1 results. J Nurs Manag. 2014;22(4):506-18.

10. Sinclair $S$, Raffin-Bouchal $S$, Venturato $L$, et al. Compassion fatigue: a metanarrative review of the healthcare literature. Int J Nurs Stud. 2017;69:9-24.

11. Tricco AC, Lillie E, Zarin W, et al. PRISMA extension for scoping reviews (PRIS MA-SCR): checklist and explanation. Ann Intern Med. 2018;169(7):467-73.

12. Khalil H, Peters M, Godfrey CM, et al. An evidence-based approach to scoping reviews. Worldviews Evid Based Nurs. 2016;13(2):118-23.

13. Levac D, Colquhoun H, O'Brien KK. Scoping studies: advancing the methodology. Implement Sci. 2010;5(69):1-9.

14. Arksey H, O'Malley L. Scoping studies: towards a methodological framework. Int J Soc Res Methodol. 2005;8(1):19-32.

15. Mellor C, Hain R. Paediatric palliative care: not so different from adult palliative care? Br J Hosp Med (2005). 2010;71(1):36-9.

16. Barnett MD, Ruiz IA. Psychological distress and compassion fatigue among hospice nurses: the mediating role of self-esteem and negative affect. $J$ Palliat Med. 2018;21(10):1504-6.

17. Bessen S, Jain RH, Brooks WB, Mishra M. "Sharing in hopes and worries"-a qualitative analysis of the delivery of compassionate care in palliative care and oncology at end of life. Int J Qual Stud Health Well-Being. 2019;14(1): 1622355. 
18. Frey R, Robinson J, Wong C, Gott M. Burnout, compassion fatigue and psychological capital: findings from a survey of nurses delivering palliative care. Appl Nurs Res. 2018;43:1-9.

19. Galiana L, Arena F, Oliver A, et al. Compassion satisfaction, compassion fatigue, and burnout in Spain and Brazil: ProQOL validation and Crosscultural diagnosis. J Pain Symptom Manag. 2017;53(3):598-604.

20. Heeter $C$, Lehto R, Allbritton M, et al. Effects of a technology-assisted meditation program on healthcare providers', interoceptive awareness, compassion fatigue, and burnout. J Hospice Palliat Nurs. 2017;19(4):314-22.

21. Hilliard RE. The effect of music therapy sessions on compassion fatigue and team building of professional hospice caregivers. Arts Psychother. 2006; 33(5):395-401.

22. Hotchkiss JT. Mindful self-care and secondary traumatic stress mediate a relationship between compassion satisfaction and burnout risk among hospice care professionals. Am J Hosp Palliat Care. 2018:35(8):1099-108.

23. Kaur A, Sharma MP, Chaturvedi SK. Professional quality of life among professional care providers at Cancer palliative care centers in Bengaluru, India. Indian J Palliat Care. 2018;24(2):167-72.

24. Melvin CS. Professional compassion fatigue: what is the true cost of nurses caring for the dying? Int J Palliat Nurs. 2012;18(12):606-11.

25. Montross-Thomas LP, Scheiber C, Meier EA, et al. Personally meaningful rituals: a way to increase compassion and decrease burnout among hospice staff and volunteers. J Palliat Med. 2016;19(10):1043-50

26. Mota Vargas R, Mahtani-Chugani V, Solano Pallero M, et al. The transformation process for palliative care professionals: the metamorphosis, a qualitative research study. Palliat Med. 2016:30(2):161-70.

27. O'Mahony S, Ziadni M, Hoerger M, et al. Compassion fatigue among palliative care clinicians: findings on personality factors and years of service. Am J Hosp Palliat Care. 2018;35(2):343-7.

28. Samson T, Shvartzman P. Secondary traumatization and proneness to dissociation among palliative care workers: a Cross-sectional study. J Pain Symptom Manag. 2018;56(2):245-51.

29. Samson T, Shvartzman P. Association between level of exposure to death and dying and professional quality of life among palliative care workers. Palliat Support Care. 2018;16(4):442-51.

30. Samson T, Lecovich E, Shvartzman P. Psychometric characteristics of the Hebrew version of the professional quality-of-life scale. J Pain Symptom Manag. 2016;52(4):575-81.

31. Sansó N, Galiana L, Oliver A, et al. Palliative care Professionals' inner life: exploring the relationships among awareness, self-care, and compassion satisfaction and fatigue, burnout, and coping with death. J Pain Symptom Manag. 2015;50(2):200-7.

32. Slocum-Gori S, Hemsworth D, Chan WY, et al. Understanding compassion satisfaction, compassion fatigue and burnout: a survey of the hospice palliative care workforce. Palliat Med. 2013;27(2):172-8.

33. Zambrano SC, Chur-Hansen A, Crawford GB. The experiences, coping mechanisms, and impact of death and dying on palliative medicine specialists. Palliat Support Care. 2014;12(4):309-16.

34. Hill RC, Dempster M, Donnelly M, et al. Improving the wellbeing of staff who work in palliative care settings: a systematic review of psychosocial interventions. Palliat Med. 2016;30(9):825-33.

35. Najjar N, Davis LW, Beck-Coon K, et al. Compassion fatigue: a review of the research to date and relevance to cancer-care providers. J Health Psychol. 2009;14(2):267-77.

36. Cross LA. Compassion fatigue in palliative care nursing: a concept analysis. J Hosp Palliat Nurs. 2019;21(1):21-8.

37. Brito-Pons G, Librada-Flores S. Compassion in palliative care: a review. Curr Opin Support Palliat Care. 2018;12(4):472-9.

38. Flarity K, Gentry JE, Mesnikoff N. The effectiveness of an educational program on preventing and treating compassion fatigue in emergency nurses. Adv Emerg Nurs J. 2013:35(3):247-58.

39. Delaney MC. Caring for the caregivers: Evaluation of the effect of an eightweek pilot mindful self-compassion (MSC) training program on nurses' compassion fatigue and resilience. PloS One. 2018;13(11):0207261.

40. Gentry JE, Baggerly J, Baranowsky A. Training-as-treatment: effectiveness of the certified compassion fatigue specialist training. Int J Emerg Mental Health. 2004;6(3):147-55.

\section{Publisher's Note}

Springer Nature remains neutral with regard to jurisdictional claims in published maps and institutional affiliations.

\section{Ready to submit your research? Choose BMC and benefit from:}

- fast, convenient online submission

- thorough peer review by experienced researchers in your field

- rapid publication on acceptance

- support for research data, including large and complex data types

- gold Open Access which fosters wider collaboration and increased citations

- maximum visibility for your research: over $100 \mathrm{M}$ website views per year

At BMC, research is always in progress.

Learn more biomedcentral.com/submissions 